\title{
Neue und wirksame Option zur Gewichtsreduktion
}

In Deutschland nehmen Übergewicht und Adipositas weiter zu, bei Frauen mehr als bei Männern, und damit verbunden ist ein höheres Morbiditäts- und Mortalitätsrisiko. Mit zunehmendem BMI nimmt die Lebenserwartung ab und auch die Gefahr für bestimmte Krebsarten steigt. Viele Menschen sind sich dieser Risiken für ihre Gesundheit gar nicht bewusst, erklärte Prof. Andreas Pfeiffer, Berlin, auf einer Fachpresseveranstaltung, dabei kann bereits eine geringe Gewichtsreduktion das Mortalitätsrisiko erheblich senken. Zwar gelingt es vielen Betroffen Gewicht abzunehmen, aber den einmal erreichten Gewichtsverlust aufrecht halten, können nur wenige. Der Grund sind metabolische Veränderungen, so PD Dr. Jens Aberle, Hamburg, mit denen der Körper auf verringerte Nahrungszufuhr reagiert. So wird bei Diäten vermehrt das appetitanregende Hormon Ghrelin ausgeschüttet. Untersuchungen haben gezeigt, dass der
Ghrelinspiegel in einer Diätphase nach 10 Wochen deutlich über seinen Normalwerten liegt und auch nach einem Jahr noch nicht den Ausgangswert erreicht hat. Ein Grund dafür, dass viele Abnehmwillige wieder an Gewicht zulegen.

Liraglutid $3 \mathrm{mg}$ wurde im März 2015 als bisher einziger GLP-1-Rezeptorantagonist in der EU zur Behandlung der Adipositas zugelassen. Die Zulassung beruht auf den Ergebnissen des umfassenden Studienkonzepts SCALE ${ }^{\mathrm{TM}}$. In 4 Studien induzierte Liraglutid $3 \mathrm{mg}$, in Ergänzung zu Diät und körperlichen Aktivitäten, einen signifikanten Gewichtsverlust von durchschnittlich $8 \%$ nach 56 Wochen und zwar unabhängig vom Prädiabetes-Status und der BMI-Kategorie, so Prof. Matthias Blüher, Leipzig. Insgesamt verloren 2/3 der Teilnehmer mit dem Medikament mindestens 5\% ihres Ausgangsgewichts, jeder Dritte sogar mehr als $10 \%$. Zudem war die Anwendung von Liraglutid $3 \mathrm{mg}$ $\left(\right.$ Saxenda ${ }^{\circledR}$ ) mit signifikanten Verbesserungen von glykämischen Parametern, Blutdruck, Biomarkern, kardiovaskulärer Risikofaktoren sowie Parametern der Lebensqualität verbunden.

Wurde Liraglutid $3 \mathrm{mg}$ ergänzend zu einer bereits deutlichen Gewichtsabnahme, im Mittel $6 \%$, eingenommen, so die Ergebnisse der Maintenance-Studie, kam es danach zu einer zusätzlichen Gewichtsabnahme in der gesamten Studienpopulation von weiteren $6,2 \%$ nach 56 Wochen. Etwa $81 \%$ dieser Patienten hielten die Gewichtsreduktion dabei vom Zeitpunkt der Einführungsphase an aufrecht.

Richard Kessing, Zeiskam

Quelle: Fachpresseveranstaltung:

„Adipositas behandeln: Eine neue Perspektive der Gewichtsreduktion mit Saxenda " am

22.3.2016 in Frankfurt. Veranstalter:

Novo Nordisk Pharma GmbH, Mainz

\section{Exakte Blutzucker-Dokumentation via Bluetooth}

Bei Anwendung des neuen ersten InsulinPens mit einer Bluetooth-Schnittstelle müssen sich die Ärzte nicht mehr auf möglicherweise ungenügend dokumentierte Blutzuckerdaten ihrer Patienten verlassen. Denn die telemedizinische Technologie ermöglicht durch die Übertragung von Blutzuckerwerten, Insulinund Broteinheiten an ein Onlineportal eine genaue Dokumentation. Wie eine Anwendungsbeobachtung zeigte, konnte mithilfe des Pens eine Absenkung der $\mathrm{HbA}_{1 \mathrm{c}}$-Werte im Mittel um 0,9\%-Punkte erreicht werden.

\section{Vollautomatisches Produktsystem}

Das vollautomatische Produktsystem überträgt die gespritzten Insulineinheiten, gemessenen Blutzuckerwerte und eingegebene Broteinheiten automatisch via Bluetooth von den Geräten ESYSTA ${ }^{\circledR}$ Pen und Lab (Blutzuckermessgerät) an das ESYSTA ${ }^{\circledR}$ Portal (www.esysta.com). Die Daten werden dort in Grafiken und Tabellen dargestellt und können über In- ternetbrowser oder die ESYSTA ${ }^{\circledR}$ App auf dem Smartphone oder Tablet eingesehen werden. Das System erfüllt die Anforderungen an ein Medizinprodukt nach CE-Norm und den höchsten IT-Sicherheitsstandard, betonte Dr. Janko Schildt, Potsdam. Der Zugriff auf die Daten ist nur durch den Patienten selbst und den von ihm autorisierten Personen wie dem Arzt oder Pflegekräften möglich.

„Mit dem digitalisierten Diabetes-Management kann eine Verbesserung der Insulineinstellung erreicht werden“, sagte Prof. Klaus Kusterer, Mannheim. Denn durch die Dokumentation der Werte aus bis zu 1000 Datensätzen erhält der Arzt eine lückenlose Auskunft über die Stoffwechsellage seiner Diabetes-Patienten. In der Darstellung werden Therapiefehler sofort sichtbar gemacht, beispielsweise wenn die Injektion von Basal- oder Bolusinsulin vergessen wurde. Algorithmen erfassen Therapiefehler und zeigen kritische Werte in einem Ampelsystem in rot an.
Eine verbesserte Insulineinstellung zeigte die Anwendungsbeobachtung „ESYSTA ${ }^{\circledR}$ S-T-A-R-T Projekt“. Darin wurde das System in Zusammenarbeit mit einer Krankenkasse und der TU Dresden im Praxisalltag an über 250 Patienten mit Insulintherapie getestet. Im Ergebnis konnte bei Typ-1- und -2-Diabetikern eine Absenkung des $\mathrm{HbA}_{1 \mathrm{c}}$-Wertes um im Mittel 0,9\%-Punkte erreicht werden, bei Typ-2Diabetikern sogar um 1,2\%. Trotz der Absenkung der $\mathrm{HbA}_{1 \mathrm{c}}$-Werte wurde keine erhöhte Hypoglykämierate beobachtet, so Kusterer weiter.

\section{Fazit}

Mit dem ESYSTA ${ }^{\circledR}$ Pen können alle gängigen Insulinpatronen verschiedener Hersteller verwendet werden, wozu als Zubehör ein Adapter erhältlich ist. Die Komponenten sind für die Patienten per Rezept verfügbar. Dr. Ralph Hausmann, Frankfurt

Quelle: Launch-Pressekonferenz: „Weltweit erster Bluetooth Insulin-Pen“, 15. März 2016, Frankfurt. Veranstalter: Emperra GmbH 